\title{
Nota Bene
}

This book is for musicians and art lovers who may have had little exposure to math, physics, and biology. It is written so that you can understand everything with no math beyond grade-school multiplication and division.

This is not a "pop science" book to be absorbed in a single reading: read a chapter that you are interested in, absorb what you can, and when you reread it, you will understand more. Honestly, I have been a professional scientist for three decades and still need to learn and relearn many of the basic concepts in these pages.

To some extent, math and science are foreign languages, and it is best to learn little by little: some topics required humanity centuries to comprehend, and particularly toward the end of the book, there remains far more to be discovered.

For ambitious readers, the "Math Boxes" use simple math to go a bit further and can be safely ignored by readers without losing the flow. Even these require only multiplication and division.

The "Sidebars" are tangential remarks. 

MUSIC, MATH, AND MIND 
